7th International Workshop on Astronomy and

Relativistic Astrophysics (IWARA 2016)

International Journal of Modern Physics: Conference Series

Vol. 45 (2017) 1760037 (4 pages)

(C) The Author(s)

DOI: $10.1142 / S 2010194517600370$

\title{
Braking Indices of Pulsars Obtained in the Presence of an Effective Force
}

\author{
Nadja S. Magalhães, André S. Okada \\ Departamento de Física \\ Universidade Federal de São Paulo (UNIFESP) \\ Rua São Nicolau 210, 09913-030 Diadema, SP, Brazil \\ nadjasm@gmail.com \\ Carlos Frajuca \\ Instituto Federal de Educação, Ciência e Tecnologia de São Paulo (IFSP) \\ R. Pedro Vicente 625, 01109-010 São Paulo, SP, Brazil \\ frajuca@gmail.com
}

Published 15 August 2017

\begin{abstract}
The theoretical calculation of braking indices of pulsars is still an open problem. In this work we present a study on this issue which adapts the model that assumes that pulsars are rotating magnetic dipoles by introducing a compensating component in the energy conservation equation of the system. Such component relates to an effective force that varies with the first power of the tangential velocity of the pulsar's crust. We tested the proposed model using data available .
\end{abstract}

Keywords: Pulsars, Stars, Star Rotation, Magnetic Fields.

PACS numbers: 9760Gb, 9835Df, 9835Eg

\section{Introduction}

Pulsars are normally modeled as rapidly rotating stars. It has been observed that the rotation frequencies of pulsars are decaying, this spin-down being quantified by the braking index (BI), $n$, defined by:

$$
n \equiv \frac{\Omega \ddot{\Omega}}{\dot{\Omega}^{2}},
$$

where $\Omega$ is the pulsar's angular velocity and the dot denotes a time derivative. According to a widely accepted model, which we will refer to as canonical, pulsars are also highly magnetized stars composed mainly of neutrons, and the main

This is an Open Access article published by World Scientific Publishing Company. It is distributed under the terms of the Creative Commons Attribution 4.0 (CC-BY) License. Further distribution of this work is permitted, provided the original work is properly cited. 
time-varying field responsible for the loss of rotational energy in a pulsar is a magnetic dipole field ${ }^{1}$. Also, the canonical model predicts $n=3$ for all existing pulsars. However, this model fails to yield the observed BI, as is shown in Table 1. Therefore, improvements on this model have been tried involving different theoretical approaches 2,3 .

Table 1. Angular velocity $(\Omega)$, its first time derivative $(\dot{\Omega})$ and braking index $(n)$ for our sample of pulsars. The constant $b$ is a result of this work, as explained in the text.

\begin{tabular}{cccccc}
\hline Pulsar & $\begin{array}{c}\Omega \\
\left(\mathrm{rad} \mathrm{s}^{-1}\right)\end{array}$ & $\begin{array}{c}\dot{\Omega} \\
\left(\times 10^{-10} \mathrm{rad} \mathrm{s}^{-2}\right)\end{array}$ & $\begin{array}{c}\ddot{\Omega} \\
\left(\times 10^{-21} \mathrm{rad} \mathrm{s}^{-3}\right)\end{array}$ & $\mathrm{n}$ & $\begin{array}{c}b \times 10^{19} \\
\left(\mathrm{~kg} \mathrm{~s}^{-1}\right)\end{array}$ \\
\hline B 0531+21 & $189.912022^{4}$ & $-24.2674^{4}$ & $78.075^{4}$ & $2.51^{5}$ & 2.9678 \\
B 0833-45 & $70.4^{6}$ & $-0.986^{6}$ & $0.19^{6}$ & $1.4^{5}$ & 0.096714 \\
B 0540-69 & $124.623817^{7}$ & $-11.8365^{7}$ & $25.6^{7}$ & $2.28^{5}$ & 1.2378 \\
B 1509-58 & 7.22613 & $41.68013054^{8}$ & $-4.24618765^{8}$ & $12.2944^{8}$ & $2.839^{5}$ \\
J 1846-0258 & $19.340994108^{8}$ & $-4.21955^{8}$ & $24.3^{8}$ & $2.64^{5}$ & 6.81415 \\
J 1119-6127 & $15.401361301^{9}$ & $-1.517708^{9}$ & $4.014^{9}$ & $2.684^{5}$ & 3.49358 \\
J 1734-3333 & $5.37327178^{10}$ & $-0.104742^{10}$ & $0.018^{10}$ & $0.99^{5}$ & 0.10201 \\
\hline
\end{tabular}

In this work we sumarize the study on a modification of the canonical model aiming at theoretically obtaining BI of pulsars that have already been observed. The modification introduces a compensating component in the energy conservation equation of the system that would correspond to a force that varies with the first power of the tangential velocity of the pulsar's crust. The full study was published elsewhere $^{11}$.

\section{Basics of the Canonical Model}

In the canonical model the radiation emitted by a pulsar derives from the magnetic energy $\left(E_{\text {mag }}\right)$, which is considered to originate exclusively from the rotational kinetic energy $\left(E_{\text {rot }}\right)$ of the neutron star.

In this model the following are constants: $B_{P}$ is the magnetic field at the pole, $R$ is the radius of the pulsar and $\alpha$ is the angle between the magnetic dipole axis and the rotation axis. The angular velocity of the pulsar, $\Omega$, varies with time. The equation for the time-averaged radiated is

$$
\dot{E}_{m a g}=\frac{1}{6 c^{3}} B_{P}^{2} R^{6} \Omega^{4} \sin ^{2} \alpha
$$

where $c$ is the speed of light in vacuum.

Energy conservation implies

$$
\dot{E}_{r o t}=-\dot{E}_{m a g}
$$

which can be put in the form

$$
\dot{\Omega}=-K \Omega^{3},
$$


where

$$
K \equiv \frac{2 m_{\perp}^{2}}{3 c^{3} I},
$$

is a constant, with $m_{\perp} \equiv \frac{B_{P} R^{3}}{2} \sin \alpha$ and $I=(2 / 5) M R^{2}$ being the moment of inertia of a solid sphere. Therefore, the canonical model predicts a gradual slowdown of the star's rotation, as is observed, but at a constant $n=3$.

\section{Modifying the Canonical Model}

For the pulsars in Table 1 the canonical model is modeling a rotational energy dissipation larger than it actually is. Therefore, in the overall power balance in (3) an additional component may be assumed. In this study we add the following effective force to the system corresponding to such component:

$$
\vec{F}=b \vec{v}
$$

where $b$ is a positive constant and $\vec{v}$ is the tangential velocity of the pulsar's surface (at the radius $r=R$ ). We are assuming that the canonical model is basically correct except for a still unknown physical context mathematically modeled by such effective force.

The power associated to this work, $\dot{W}$, is then

$$
\dot{W}=b R^{2}\left(\Omega^{2}+\dot{\Omega} \phi\right),
$$

which we include in (3), yielding

$$
I \Omega \dot{\Omega}=-b R^{2}\left(\Omega^{2}+\dot{\Omega} \phi\right)-K I \Omega^{4} .
$$

The expression for the BI in this model can be found differentiating (7) with respect to time and then isolating $\ddot{\Omega}$, yielding, after some algebra,

$$
n=3+\left(K \Omega^{3}+\dot{\Omega}\right)\left(K \Omega^{3}+\frac{b R^{2} \Omega}{I}\right)^{-1} .
$$

The expression for the constant $b$ as a function of the BI is obtained inverting (8):

$$
b=\frac{K I \Omega^{2}}{R^{2}}+\left(-I \dot{\Omega}-K I \Omega^{3}\right)\left((n-3) \Omega R^{2}\right)^{-1} .
$$

We applied (9) to the pulsars in Table 1 and obtained the values for $b$ presented in that table, assuming: a typical mass value of ${ }^{12} M=1.4 M_{\odot}$, where $M_{\odot}$ represents one solar mass; the usual value of $R=10^{4} \mathrm{~m} ; \sin ^{2} \alpha=1$ to simplify the calculations; a typical value of the magnetic field at the poles based on the canonical model: $B_{P}=10^{12} \mathrm{G}$. We found that $b$ is mainly of the order of $10^{19} \mathrm{~kg} \mathrm{~s}^{-1}$. 


\section{Conclusions}

We proposed a modification in the canonical model for pulsars' spin-down which corresponds to an effective force that would be tangential to the star's rotation motion, involving all yet unknown physical contributions that make pulsars' BI less than three.

As a first result of this work we found that the proportionality constant, $b$, of the tangential force is typically of the order of of $10^{19} \mathrm{~kg} \mathrm{~s}^{-1}$. This high value is expected to simplify the expression for the braking index that enables the prediction of this index for other pulsars. The investigation continues in a more lengthy paper ${ }^{11}$.

\section{Acknowledgments}

The authors acknowledge the Brazilian federal funding agency $\mathrm{CNPq}$ for financial support (grants 309295/2009-2, 149107/2010-2 and 312906/2013-7, respectively) as well as the National Institute of Science and Technology in Astrophysics (INCT-A, Brazil). NSM and CF acknowledge FAPESP for support under the thematic project \# 2013/26258-4.

\section{References}

1. J. P. Ostriker and J. E. Gunn, ApJ 157, 1395 (1969).

2. N. S. Magalhaes, T. A. Miranda, and C. Frajuca, ApJ, 755, 54 (2012).

3. F. F. Kou , H. Tong, MNRAS, 450, 1990 (2015).

4. A. Lyne, R. Pritchard, and F. Graham Smith, MNRAS 265, 1003 (1993).

5. R. N. Manchester, G. B. Hobbs, and A. Teoh et al., ApJ 129, 1993 (2005). ATNF Pulsar Catalogue webpage: www.atnf.csiro.au. Access in 09/19/2016.

6. A. Lyne, R. Pritchard, and F. Graham Smith et al., Nature 381, 497 (1996).

7. P. T. Boyd, G. W. van Citters, and J. F. Dolan et al., ApJ 448, 365 (1995).

8. M. A. Livingstone, V. M. Kaspi, and F. P. Gavriil et al. Ap\& $S S$ 308, 317 (2007).

9. P. Weltevrede, S. Johnston, and C. M. Espinoza, MNRAS 411, 1917 (2011).

10. C. M. Espinoza, A. G. Lyne, and M. Kramer et al. ApJ 741, L13 (2011).

11. N. S. Magalhaes, A. S. Okada, and C. Frajuca, MNRAS, 461, 3993 (2016).

12. J. M. Lattimer and M. Prakash, Science, 304, 536 (2004). 